\title{
REJECTION OF REPEATABLE RUN-OUT IN HDDS USING INVERSE FREQUENCY DYNAMICS
}

\author{
Motohiro Kawafuku* Makoto Iwasaki* \\ Hiromu Hirai * Atsushi Okuyama ** \\ * Department of Electrical and Computer Engineering \\ Nagoya Institute of Technology, Nagoya, Japan \\ ${ }^{* *}$ Hitachi STRC
}

\begin{abstract}
In order to suppress the Repeatable Run-Out (RRO) of a following control system in HDDs is quite effective to increase the disk density. Many approaches were presented in former literatures, e.g. by applying adaptive filters and/or repetitive controllers. These approaches, however, require the complicated design in structures and parameters, preventing the controller design from saving time and labor. This paper presents a novel algorithm to suppress the effects of RRO on following control performance. In the proposed algorithm, the FFT (Fast Fourier Transform) is applied to identify a frequency characteristic of RRO. Based on the identified characteristics, an inverse system in frequency domain can be achieved to reject the effect of RRO. Numerical simulations and experimental results show the effectiveness of the proposed control. Copyright (c)2005 IFAC
\end{abstract}

Keywords: Accuracy, Compensators, Control algorithms, Disturbance rejection, Fast Fourier transforms, Frequency signal analysis, Inverse system

\section{INTRODUCTION}

The capacity of hard disk drive has increased significantly in recent years, while the size of disk drive is keeping smaller. With the current areal density growth rate of more than $40 \sim$ $60 \%$ per year, the bit sizes in disk drives are decreasing rapidly. The areal density is defined as a multiplication of track density and linear density. The industry is currently manufacturing products with track density at over 10000 trackper-inch (TPI). With rapid increase in TPI, in track-following mode, the head positioning servo controller must be precisely performed on the desired track against various disturbances caused by the dynamics of mechanical systems. In the track following, the non-circular track path and the eccentricity of the spindle will cause the repeatable run-out (RRO), and the disk vibration and bearing defect will cause the non-repeatable run-out (NRRO).

In order to achieve the maximum feasible TPI for the given media and head, the track misregistration (TMR) budget should be carefully estimated. TMR is composed of many factors such as the width of the head read/write element, NRRO of a spindle motor, head movements during external shock, and vibrations. In order to reduce these shock/vibration responses, the bandwidth of a disk drive servo system should be expanded. However, the expansion of the bandwidth is limited by possible sampling frequency and mechanical resonance of a head-disk assembly (HDA). The following control of HDDs requires the precise positioning and robustness against several disturbances. The result of the numerical analysis is shown that RRO is effected by all disturbance. 


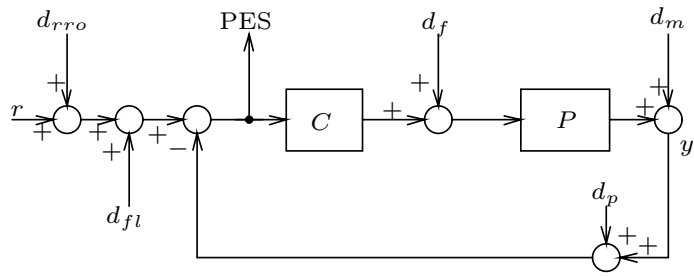

Fig. 1. Block Diagram of HDDs servo system.

Considering this result, it is important to reduce the effects of RRO in precisely control of the following control system. In this research, the inverse system compensator based on frequency analysis is applied to reject the effects of RRO.

\section{SYSTEM MODELING OF HDDS}

\subsection{Identification of plant mechanism}

Figure 1 shows a simplified block diagram of a disk drive servo system. In this figure, $C$ is a controller, $P$ is a plant model of the disk drive servo system, PES is the track following Position Error Signal, $d_{\text {rro }}$ is the disturbance due to the repeatable runout $(\mathrm{RRO}), d_{f l}$ is disk flutter vibrations, $d_{f}$ is the force disturbance due to external shock and vibrations, $d_{m}$ is the position-mode disturbance includes mechanical vibrations, $d_{p}$ is the positionmode disturbance due to electrical noise such as media noise, servo demodulator noise, etc. The goal of the servo system is to make the system output exactly follow the input signal $r$ by minimizing the effect of several disturbances.

The plant model $P$ consists of inertia with friction and has many mechanical resonance. The transfer function of the plant system is used as the following equation.

$$
P(s)=\frac{A}{m s^{2}+c s+k}+\sum_{r=1}^{14} \frac{K_{r}}{s^{2}+2 \zeta_{r} \Omega_{r} s+\Omega_{r}^{2}}(1)
$$

The first term of the right hand side of the Eq. (1) is expressed the inertia with friction. In this term, $m$ is the mass, $k$ is a spring constant, $c$ is a damping constant of the inertia with friction. The inertia with friction can be modeled as a secondorder system whose breakpoint frequency is about $60 \sim 100[\mathrm{~Hz}]$. The second term of the right hand side of the Eq. (1) is expressed the mechanical resonanse mode of the plant system. In this term, $K_{r}$ is a modal constant, $\zeta_{r}$ is a modal damping coefficient and $\Omega_{r}$ is an undamped natural angular frequency.

To deciding these parameter of the second term of the right hand side in the Eq. (1), the differential iteration method (Nagamatsu, 1993) that is a method for deciding accurate modal parameters by repeated calculation while all unknown modal

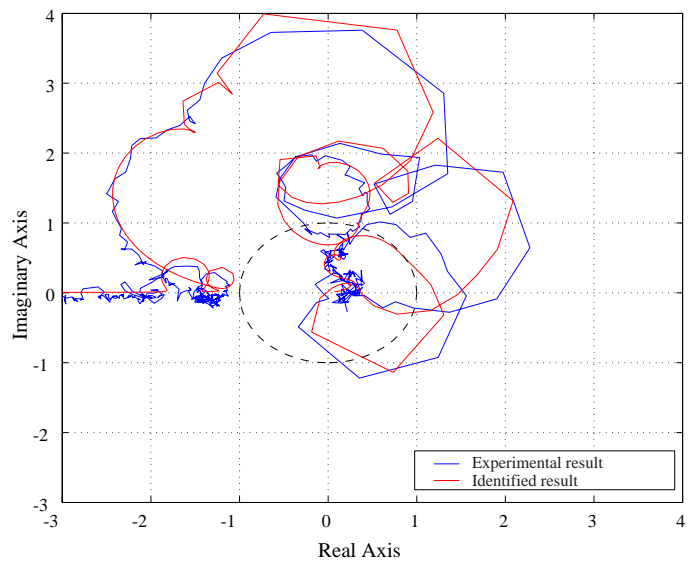

Fig. 2. Nyquist diagram of plant model.

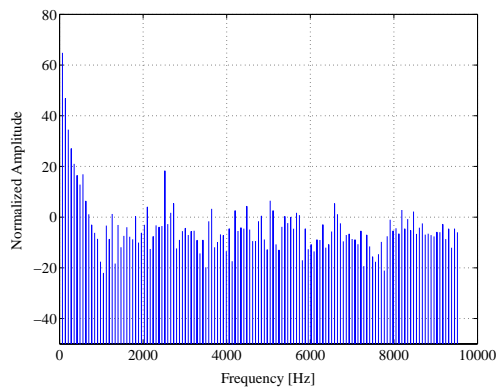

Fig. 3. Power spectrum of $d_{\text {rro }}$.

parameters are changed to the linear term was used(Kawafuku et al., 2003).

Figure 2 shows the nyquist diagram of the real plant and the identified model $P(s)$. It is shown that the frequency characteristics of the real plant and the identified model are in good agreement with each other.

\subsection{Identification of disturbances}

In the Fig. 1, the transfer function of PES is related to $r, d_{r r o}, d_{f l}, d_{f}, d_{m}$ and $d_{p}$ by the following equation:

$$
\begin{aligned}
P E S(s) & =\frac{1}{1+P(s) C(s)} R^{\prime}(s)-\frac{P(s)}{1+P(s) C(s)} D_{f}(s) \\
& -\frac{1}{1+P(s) C(s)} D_{p}(s)-\frac{1}{1+P(s) C(s)} D_{m}(s)(2)
\end{aligned}
$$

where

$$
R^{\prime}(s)=R(s)+D_{\text {rro }}(s)+D_{f l}(s) .
$$

A comprehensive identification and analysis of each disturbance component can be found in reference (Numasato et al., 1999) and (Abramovitch et al., 1998). The frequency characteristics of each disturbance is shown in Fig. $3 \sim$ Fig. 7 .

The frequency band of the characteristic of $d_{m}$ consists mainly of mechanical resonance and $d_{f l}$ is very sharp which has a large peak gain. It 


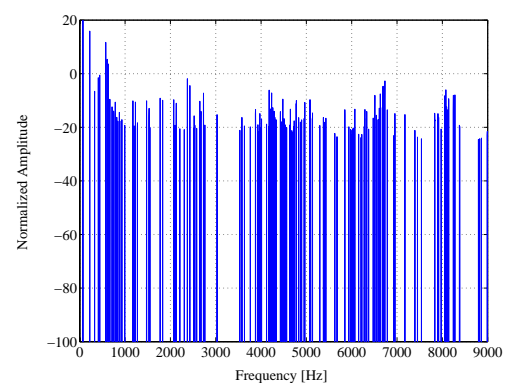

Fig. 4. Power spectrum of $d_{m}$.

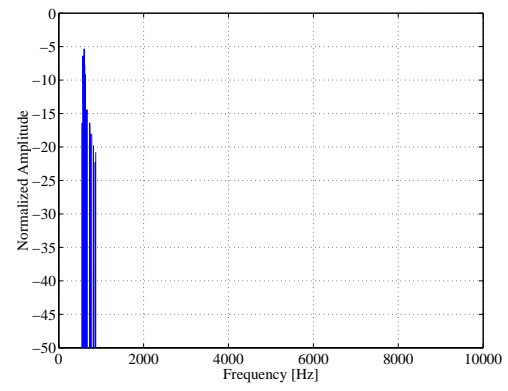

Fig. 5. Power spectrum of $d_{f l}$.

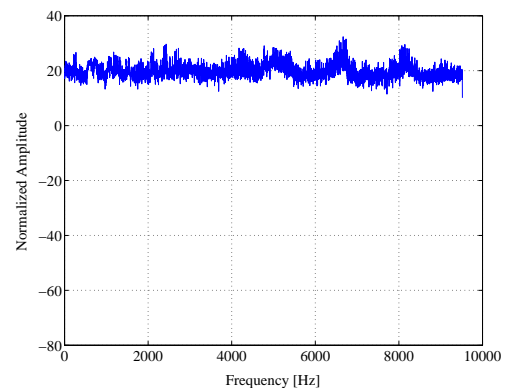

Fig. 6. Power spectrum of $d_{f}$.

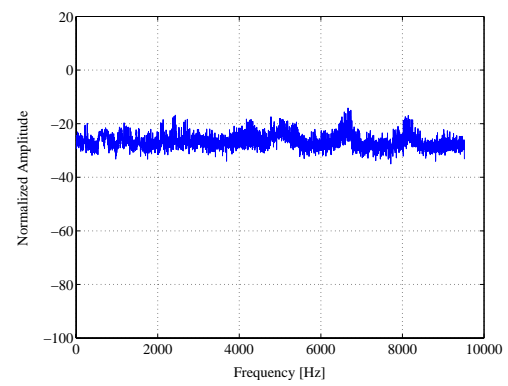

Fig. 7. Power spectrum of $d_{p}$.

is known that $d_{f}$ and $d_{p}$ are almost random signal. The frequency characteristics from these disturbances to $P E S$ can be modeled by the servo transfer function shown in Eq. (2). To using these disturbance models in order to estimate the effect for the following system in time domain, these models were transformed from frequency domain into time domain. In numerical simulation, each disturbance is inputted to the HDDs servo system shown in Fig. 1 independently, and verify the proportion for the effect of the whole disturbance in PES. The standard deviation $\pm 3 \sigma$ of each disturbance is shown in Table 1 . The result of
Table 1. Standard deviation $\pm 3 \sigma$

\begin{tabular}{|rc|c|}
\hline & & $\pm 3 \sigma[\%]$ \\
\hline Repeatable Run-Out & $d_{r r o}$ & 99.10 \\
\hline Mechanical Vibration & $d_{m}$ & 8.22 \\
\hline Flutter Vibration & $d_{f l}$ & 2.39 \\
\hline Position Disturbance & $d_{p}$ & 3.74 \\
\hline Force Disturbance & $d_{f}$ & 5.49 \\
\hline
\end{tabular}

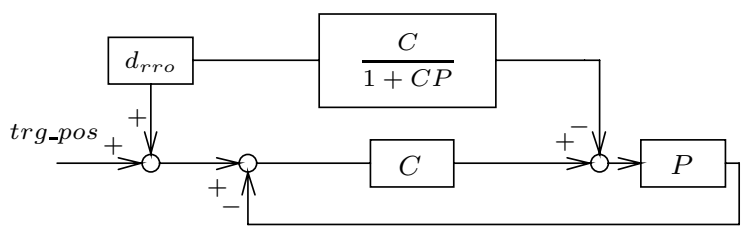

Fig. 8. Ideal rejection compensator for RRO.

the numerical analysis showed that the element of RRO has great influence in all disturbances.

\section{EFFECT OF RRO SUPPRESSION BY USING IDEAL COMPENSATOR}

Describing the previous section, the standard deviation of PES is influenced a great deal with the disturbance due to the RRO. In this section, the depressing effect of the disturbance due to the RRO by using ideal compensator is verified. The ideal compensator is creating a control signal by using the RRO signal directly. The block diagram of the following control system with ideal compensator is shown in Fig. 8. Where, $C$ is the controller, $P$ is the identified model that was indented the previous section. The servo bandwidth frequency is set at $450[\mathrm{~Hz}$. The impress point of the control signal is different from the input point of RRO. So, the control signal characteristic is transformed by the transfer function from input point of the control signal to the input point of RRO.

The simulation result by using the ideal compensator is shown in Fig. 9. The standard deviation $\pm 3 \sigma$ is shown in Table 2. From these results, if the characteristics of RRO can be analyzed before control signal impress, it is possible to reject the subtotal disturbance of RRO by using the RRO signal directly to create the control signal. However, it is impractical idea because the actual hardware has different characteristics of RRO between all tracks, and it caused to increase the memory capacity if the all RRO characteristics of the hardware are memorized in the memory device. Moreover, the characteristic of RRO cannot sense directly. In the next section, the inverse system compensator based on frequency analysis of PES is proposed and the validity of the proposed compensator is verified. 


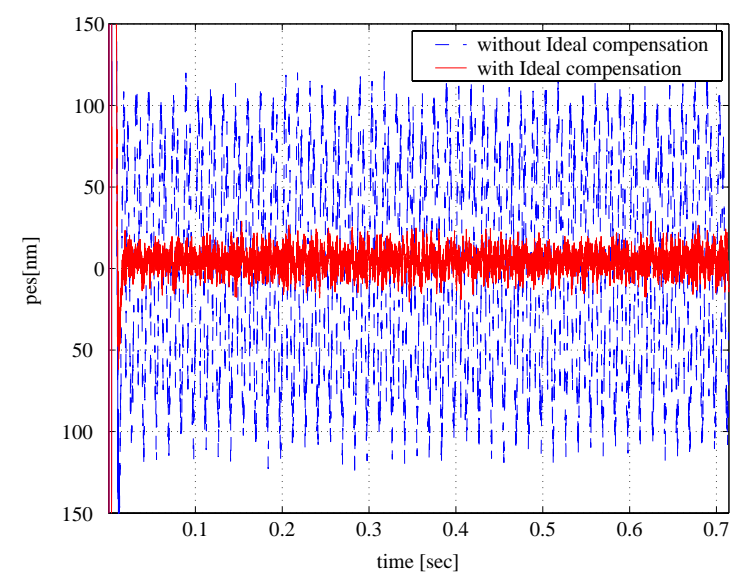

Fig. 9. Time response of RRO with/without ideal compensator.

Table 2. Standard deviation $\pm 3 \sigma$ of PES with/without ideal compensator

\begin{tabular}{|r|c|}
\hline & $\pm 3 \sigma[\%]$ \\
\hline Without Ideal Compensator & 100 \\
\hline With Ideal Compensator & 10.33 \\
\hline
\end{tabular}

Proposed Compensator

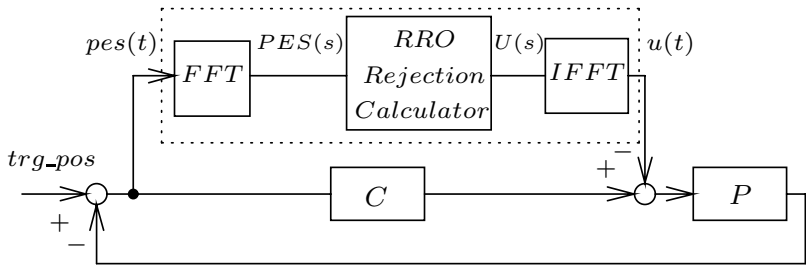

Fig. 10. Following control system with proposed controller.

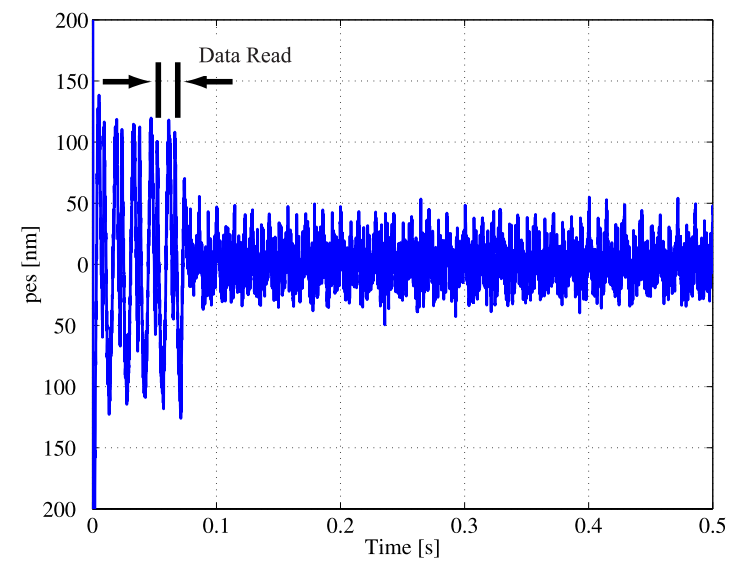

Fig. 11. Time response of pes $(t) .(\mathrm{R}=1)$

\section{INVERSE SYSTEM COMPENSATOR BASED ON FREQUENCY ANALYSIS}

The block diagram of the following control system with proposed compensator is shown in Fig. 10. Where, $C$ is the controller and the servo bandwidth frequency without proposed compensator is set at $450[\mathrm{~Hz}]$. The control signal from proposed compensator is created as following steps.

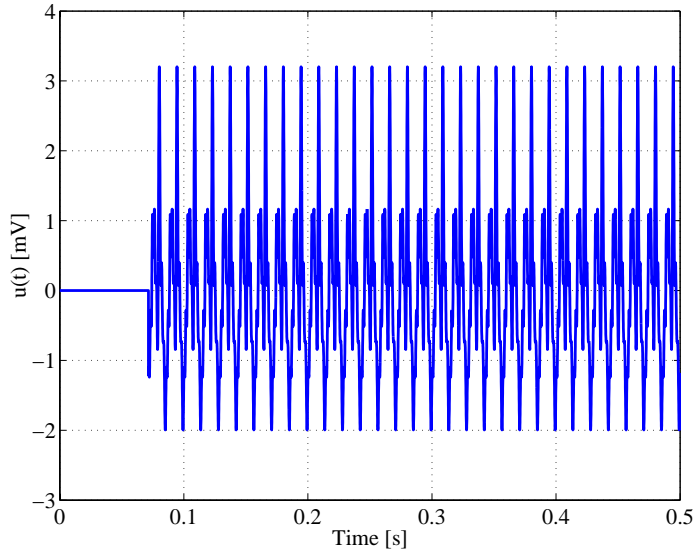

Fig. 12. Time response of $u(t) .(\mathrm{R}=1)$

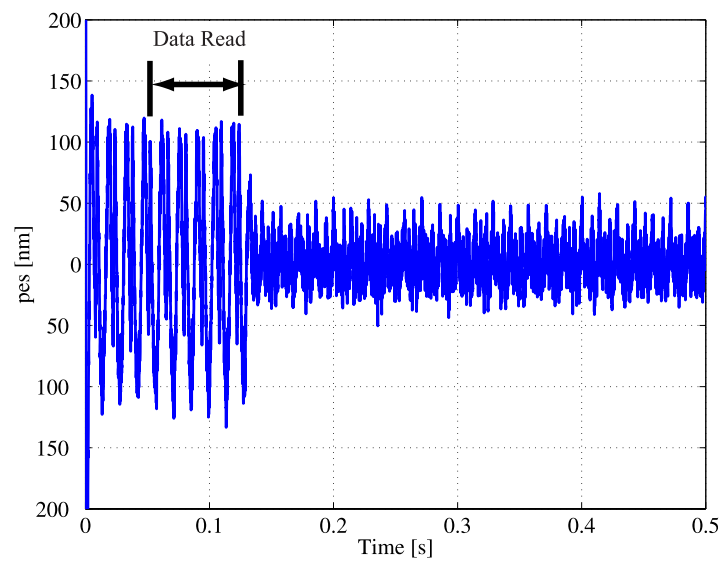

Fig. 13. Time response of pes $(t) .(\mathrm{R}=5)$

1. Store the PES data on the basis of the sector number 0 until disk rotates $R$ cycles.

2. Averaging each sector data to reduce the effect of NRRO.

3. Calculate the gain and phase values at the integer multiple frequency point of the fundamental frequency of RRO by using Fast Fourier Transformation.

4. Calculate the compensation signal characteristic at the input point by using the transfer function $\left(\frac{P}{1+C P}\right)^{-1}$.

5. Transform the compensated characteristic from frequency domain to time domain and impress the signal as $u(t)$.

Notice that the proposed controller does not construct the closed-loop system because the proposed compensator is not connected after reading the position error signal, and the impress signal of the proposed compensator is not inputted when the compensator read the PES. It is said that it does not need to consider about the stability of the closed-loop system with the proposed compensator. 


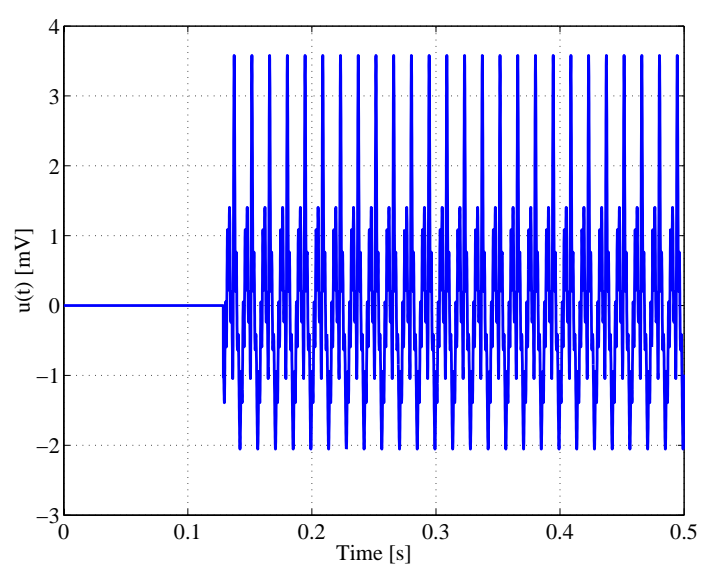

Fig. 14. Time response of $u(t) .(\mathrm{R}=5)$

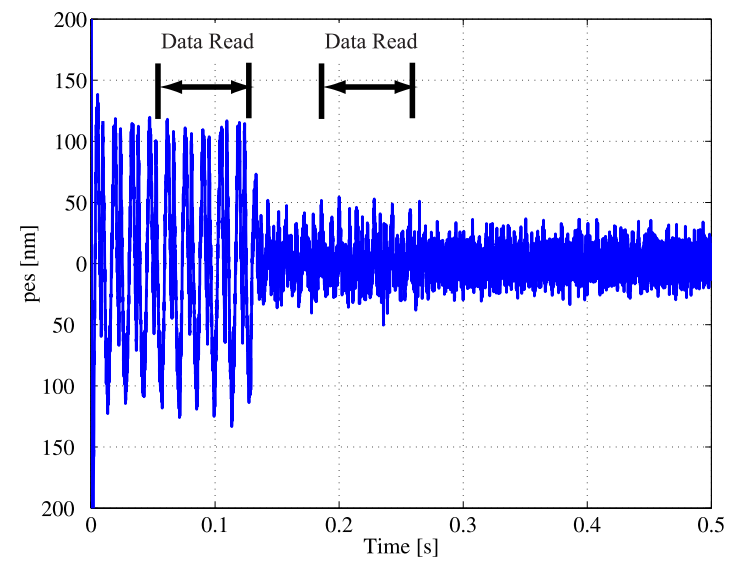

Fig. 15. Time response of pes $(t) .(R=5$, iteration $=2$ )

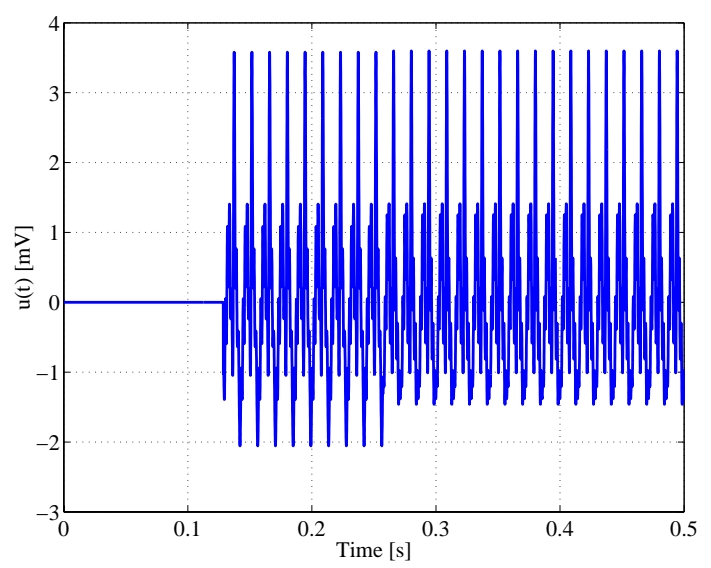

Fig. 16. Time response of $u(t) .(R=5$, iteration $=2$ )

\section{SIMULATION RESULTS}

The validity of the proposed compensator is verified by using the simulator. The fundamental frequency of the RRO of the simulator is set at $70[\mathrm{~Hz}]$. In this paper, 10 frequency points of the integer value of RRO (from 70 to $700[\mathrm{~Hz}]$ ) are picked up to reduction. Where, 2 pattern of the compensator, the difference of each compensator is the data store length: one is 1disk rotate $(R=$

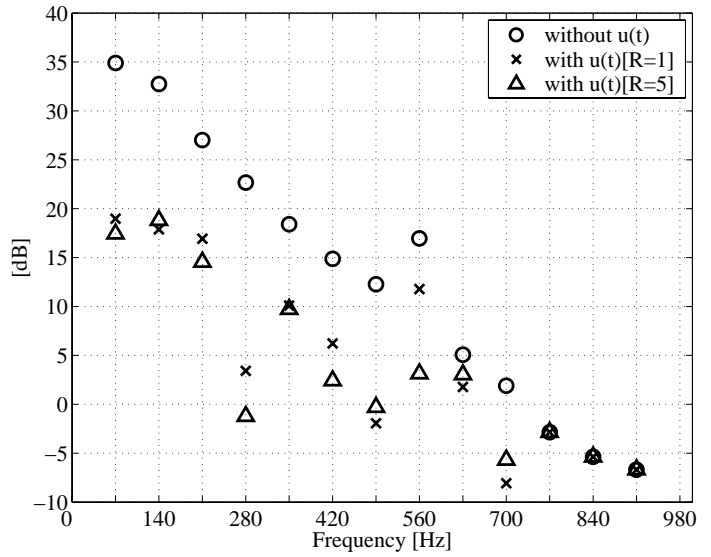

Fig. 17. Power spectrum of PES.

Table 3. Standard deviation $\pm 3 \sigma$ with proposed compensator

\begin{tabular}{|c|c|}
\hline & $\pm 3 \sigma[\%]$ \\
\hline Without Compensator & 100 \\
\hline $\mathrm{R}=1$ & 25.53 \\
\hline $\mathrm{R}=5$ & 23.83 \\
\hline $\mathrm{R}=5$, Iteration $=2$ & 17.29 \\
\hline
\end{tabular}

1) and the other one is 5 disk rotate $(R=5)$, are simulated to verified the proposed compensator. In the simulation, verification data was stored after three disk rotation to reject the transient response of the plant $P$ caused by the disturbances input. The calculation time from step 2 to step 5 dose not consider, so the control input signal is impressed to the next sampling time. At the $R=5$, the 2 times iteration compensation performance was also verified. In this time, the data that use the calculation of the 2 nd compensation signal is stored after five rotation of the disk when the first compensation signal impress the following control system.

The time responses of PES and control input signal $u(t)$ with proposed compensator is shown in Fig. 11 16. The power spectrum of PES with/without proposed compensator is shown in Fig. 17. The standard deviation $\pm 3 \sigma$ is shown in Table 3. Shown in these simulation results, it is said that the standard deviation of PES with proposed compensator $(R=1)$ is $74 \%$ and with proposed compensator $(R=5)$ is $79 \%$ less than that without the compensator. Moreover, $\pm 3 \sigma$ with duplicate compensation with iteration method is $84 \%$ less than that without compensator. Shown in Fig. 17, it is also said that the proposed compensator with data store cycle $R=5$ is more suppress the effect of RRO, especially lower frequency domain, than that with data store cycle $R=1$. The difference between each result is that the control signal accuracy for reject the effect of $\mathrm{RRO}$ is increased because of the effect of NRRO is decreased by averaging the store data of PES. 


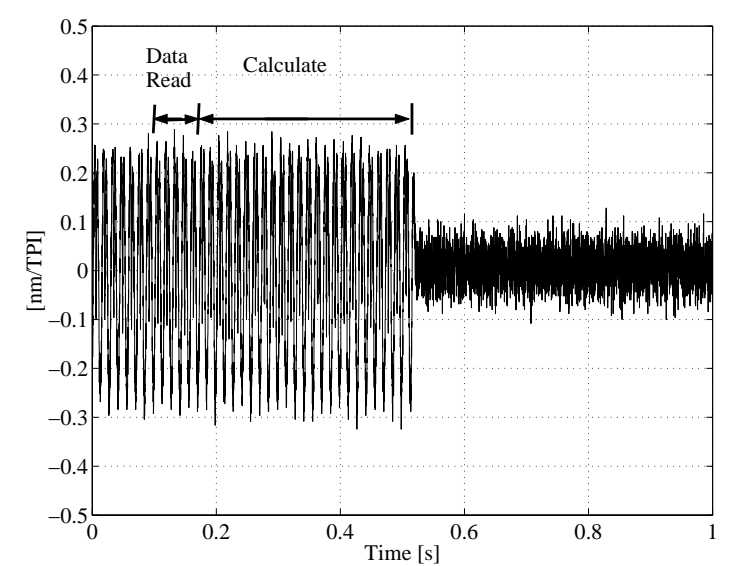

Fig. 18. Time response of pes $(t)$.

\section{EXPERIMENTAL RESULTS}

In this section, the validity of the proposed compensator is verified by experimentation. In the experimentation, the rejected number of RRO is 10 (from $70 \sim 700[\mathrm{~Hz}]$ ) and the stored cycle number of PES is $5(R=5)$. In the experimentation, because of the impossibility to define the calculation time to create the control input signal, it is impossible to get the start period to impress the control signal and it is possible to unstable of the following control system. To avoid this problem, the start period is fixed at sector number 0 . The time response of PES and power spectrum of PES with/without proposed compensator are shown in Fig. 18 and 19 respectively. Shown in Fig. 18, the amplitude of PES is decreased by suppress the effect of the 10th modes (from $70 \sim 700[\mathrm{~Hz}]$ ) RRO. Then, the standard deviation $\pm 3 \sigma$ with proposed compensator is about $80 \%$ less than that without proposed compensator. At this time, the calculation time of the proposed compensator is about 0.4 [s] (the rotation number is about 30 ). On the other hand, it need about 7.1 [s] (the rotation number is about 500) to create the precise parameter of adaptive filter (Kawafuku et al., 2004). It is said that the time to the compensation can be drastically decreased.

\section{CONCLUSION}

This paper describes that the effects of RRO on PES can be rejected by the inverse system compensator based on frequency analysis. Using proposed compensator, it is possible to reject the effect of RRO that exist in higher frequency region of the servo bandwidth of closed-loop system. Numerical simulations and experimental results show the standard deviation $\pm 3 \sigma$ with proposed compensator can be achieved about $80 \%$ less than that of without proposed compensator. The realtime processing to construct the compensator can be reduced to only $5 \%$ in processing time
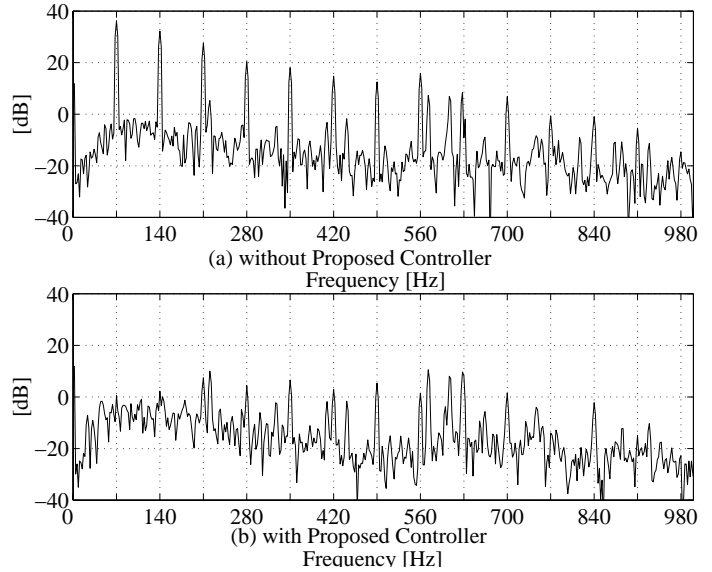

Fig. 19. Power spectrum of PES.

comparing to that of by the conventional adaptive filter. These results show the effectiveness of the proposed compensator.

\section{REFERENCES}

Abramovitch, D., T. Hurst and D. Henze (1998). An overview of the pes pareto method for decomposing baseline noise sources in hard disk position error signals. IEEE Trans. on Magnetics 34(1), 17-23.

Kawafuku, M., K. Otsu, H. Hirai and M. Kobayashi (2003). High performance control design of hdd based on precise system modeling using differential iteration method. Proc. of 2003 American Control Conference (ACC2003) pp. 4341-4346.

Kawafuku, M., M. Iwasaki, H. Hirai, M. Kobayashi and A. Okuyama (2004). Rejection of repeatable runout for hdds using adaptive filter. Proc. of Int. Workshop on Advanced Motion Control (AMC-2004) pp. 305-310.

Nagamatsu, A. (1993). Introduction to Modal Analysis (in Japanese). Corona publishing. Japan.

Numasato, H., K. Usui, Y. Hamada and T. Yamaguchi (1999). An analysis method of head positioning error for magnetic drives (an analysis method of pes in track-following mode)(in japanese). Trans. of JSME Series C 65, 3245-3251. 